\title{
A new method for human ear recognition using Haar wavelet decomposition and LDA/GSVD
}

\author{
Hailong Zhao \\ Beijing University of Civil Engineering and Architecture, Beijing 100044, China \\ zhaohailong@bucea.edu.cn
}

\begin{abstract}
Keywords: haar wavelet decomposition, LDA/GSVD, human ear recognition.
Abstract. In the case of high dimension and small sample, the feature extraction method using Fisher linear discriminant analysis has a problem of pathological singularity. There are many solutions to this problem, in which LDA/GSVD algorithm overcomes the shortcomings of the existing methods, has a good recognition rate. However, the direct use of LDA/GSVD to reduce the dimension of human ear images, it still encounters the problem of large amount of calculation and slow calculation speed. So the author proposes a new method which apply wavelet decomposition on the human ear image and then use LDA/GSVD algorithm. The method is proved by experiments that it has a good recognition rate and is an effective feature extraction approach.
\end{abstract}

\section{Introduction}

Biometrics refers to the technology of computer using personal physiological or behavioral characteristics for personal identification and authentication. It has broad application prospects in the fields of public security, financial services and network security. Biometrics used for identity authentication should be universality, uniqueness, stability, acceptability and security. Common biometrics include fingerprints, irises, face, palms, gait, voice and so on. In addition to its universal and unique features, human ears have unique advantages over traditional biometrics in that they are less susceptible to facial expressions and make-up, less damage, and consistent color distribution[1]. So ear recognition is concerned by many scholars.

Fisher Linear Discriminant (FLD), also known as Linear Discriminant Analysis (LDA), aims at the sample separability, finding a set of linear transformations that minimize the in-class divergence $S_{W}$ for each class and maximize divergence between classes $S_{B}$. The classic linear discriminant analysis uses the Fisher criterion function .

$$
J_{F}\left(W_{o p t}\right)=\underset{W}{\arg \max } \frac{\left|W^{T} S_{B} W\right|}{\left|W^{T} S_{W} W\right|}
$$

However, in pattern recognition, because of the small sample problem, the in-class divergence matrix $S_{W}$ is singular and irreversible, which makes it impossible to directly use the eigenvector Method to get the transformation matrix $W$.For solving the small sample problem, a lot of scholars have put forward many solutions, the most famous one is the Fisherface method proposed by Belhumeur et al. [2]. The basic idea of this method is to first use principal component analysis (PCA) Dimension reduction, eliminate the singularity, and then use the linear discriminant analysis method to classify.In addition to this, some scholars have proposed an LDA/GSVD algorithm to solve the singularity problem of $S_{w}$ [3] - The algorithm avoids the direct inversion of $S_{w}$ in the classical LDA algorithm by generalized singular value decomposition.

LDA/GSVD algorithm is an effective method to reduce dimensions.But If using the LDA/GSVD algorithm to directly reduce the size of the sample image, there is also a problem that when the sample image itself is large, the dimension reduction process becomes very slow or even crashes. Therefore, the author proposes a method of two-dimensional discrete wavelet decomposition of high-dimensional 
sample images and then using LDA/GSVD algorithm to reduce the dimension of low-frequency coefficients obtained after wavelet decomposition.

In this paper, a new method is proposed in this paper that combine wavelet decomposition and LDA/GSVD to extract features and NN as classifier. The remainder of the paper is organized as follows: In Section 2, the relative concepts of wavelet transform is introduced and the examples that use wavelet to decompose and reconstruct the images. In Section 3, the LDA/GSVD algorithm is introduced. In Section 4, we present the data sets and experimental results for the USTB(79) ear databases. Finally, we formulate our conclusions in Section 5.

\section{Wavelet Decomposition and Haar wavelet}

The basic idea of wavelet decomposition is to use a set of wavelet functions or basis functions to represent a function or signal, such as an image signal. In the field of signal analysis, the wavelet decomposition of the signal in the time domain depends on two parameters: the scale (or frequency) and the time. Wavelet decomposition is a tool of time-frequency localization or time-frequency localization.Fourier analysis and is widely used in signal processing, Image compression, pattern recognition, quantum physics and many non-linear. Wavelet transform in the time domain and frequency domain have good localization characteristics, is a time-frequency localization or time-frequency positioning tool [4], known as the "mathematical microscope."

Haar wavelet is discontinuous in the time domain, but it has the advantages of high speed in image decomposition and reconstruction, high compression ratio of reconstructed image and easy analysis and processing of images. Therefore, Haar wavelet is chosen as the basis function for two-dimensional discrete wavelet decomposition.

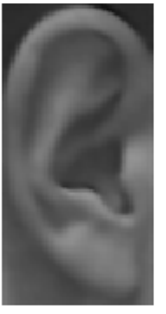

(a)

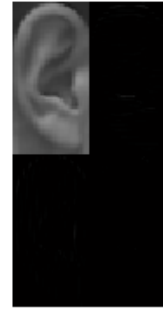

(b)

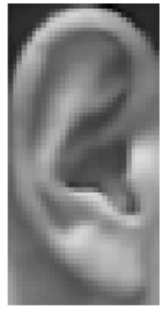

(c)

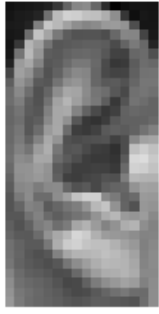

(d)

Fig.1 Sample images of wavelet decomposition and reconstruction

After the original image is decomposed by wavelet, a series of sub-images with different resolutions can be obtained. The corresponding frequencies of sub-images with different resolutions are different. Most of the points on high-resolution (ie, high-frequency) images are close to zero, the more noticeable the higher frequencies are. For an image, the representation image is mainly the low-frequency part. Therefore, in order to reduce the size of the image, we can remove the high-frequency part of the image and keep only the low-frequency part of the image. As shown in Fig.1, (a) is the original image, (b) is the low-frequency and high-frequency information of the original image after two-dimensional discrete wavelet decomposition, and (c) is the first level of decomposition of the original image, (D) is the second level of decomposition of the original image. It should be noted that in order to look more concise and intuitive, where I adjusted them to a unified size.

\section{LDA/GSVD Algorithm}

The LDA/GSVD algorithm proposed by Peg Howland et al. [3] effectively solves the ill-posed singularity problem when using the feature extraction method based on Fisher linear discriminant analysis in high dimension and small sample. The algorithm is described as follows:

For a given sample matrix $A \in i^{D \times N}$, the vector $a_{k} \in i^{D \times 1}\{k=1, \mathrm{~L}, N\}$ represents each sample vector, where $C$ is the number of samples and $N$ is the total number of samples. $N_{i}\{i=1,2, \mathrm{~L}, C\}$ represents number of each class, $m_{i}$ represents average value of each class samples and $m$ represents 
average value of all class samples. We need to find a matrix $G \in i^{D \times(C-1)}$ that reduces the dimension of the sample while keeping the class information of the sample. The objective function is $J(G)=\operatorname{Max} \operatorname{trace}\left(\left(G^{T} S_{W} G\right)^{-1} G^{T} S_{B} G\right)$

1) Calculate $H_{b}$ and $H_{w}$ 。

$$
\begin{aligned}
& H_{b}=\left[\sqrt{N_{1}}\left(m_{1}-m\right), \mathrm{L}, \sqrt{N_{C}}\left(m_{C}-m\right)\right] \in i^{D \times C} \\
& H_{w}=\left[A_{1}-m_{1} e_{1}^{T}, A_{2}-m_{2} e_{2}^{T}, \mathrm{~L}, A_{C}-m_{C} e_{C}^{T}\right] \in i^{D \times N}
\end{aligned}
$$

2) Join $H_{b}$ and $H_{w}$, formulate matrix $K=\left(\begin{array}{c}H_{b}^{T} \\ H_{w}^{T}\end{array}\right) \in i^{(C+N) \times D}$ 。

3) Apply completely orthogonal decomposition on $K[5], P^{T} K Q=\left(\begin{array}{ll}R & 0 \\ 0 & 0\end{array}\right)$ 。

4) Calculate $t=\operatorname{rank}(K)$, and set $M=P(1: C, 1: t)$ 。

5) Apply Singular value decomposition on $M, U^{T} M W=\sum_{A}$ 。

6) Calculate $X=Q\left(\begin{array}{cc}R^{-1} W & 0 \\ 0 & I\end{array}\right)$ And take the first $\mathrm{C}$ column assignment to $\mathrm{G}$ 。

7) $Y=G^{T} A, Y$ is output matrix of reduce dimensions。

\section{Experimental Procedure and Results}

The USTB(79) ear database includes 869 images from 79 persons and every person has 11 images. We can see from Figure 2: (a) is a person's frontal ear image,(b-k) are the ear images that the person rotate $5,10,15,20,25,30,35,40,45$ and 60 degrees respectively to right. The images are under the same illumination conditions.
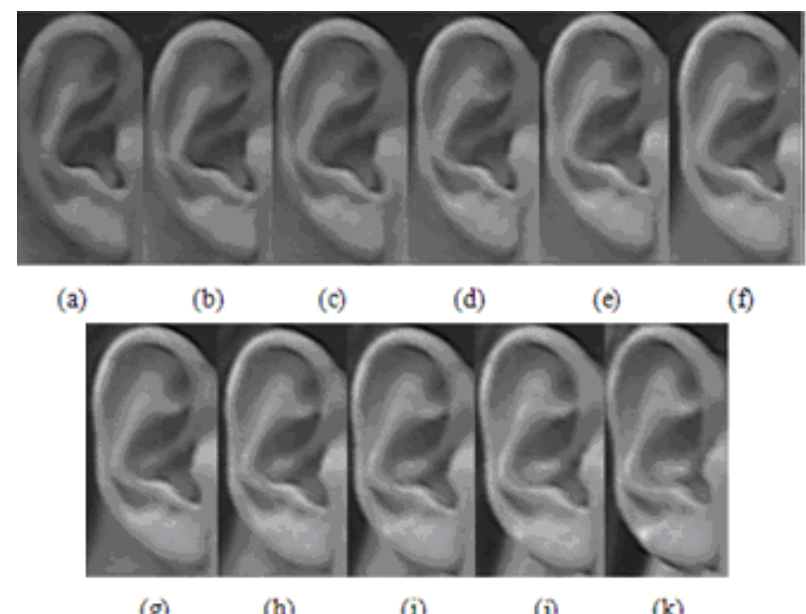

Fig. 2 Sample images from USTB(79) ear database

The experimental procedure using Haar wavelet and LDA/GSVD can be summarized as follows:

1) Pre-processing all the ear images in the database,including histogram equalization and geometry normalization. The images are resized to $80 \times 160$

2) All ear images are divided into 11 groups according to rotation degrees. When one group is selected as test set,the others are training set.

3) Apply Haar wavelet decomposition on every images.

4) Reduce dimension of the ear images which are acquired by 3) using output matrix G.

5) Use Nearest Neighbor classifier to make decision. 


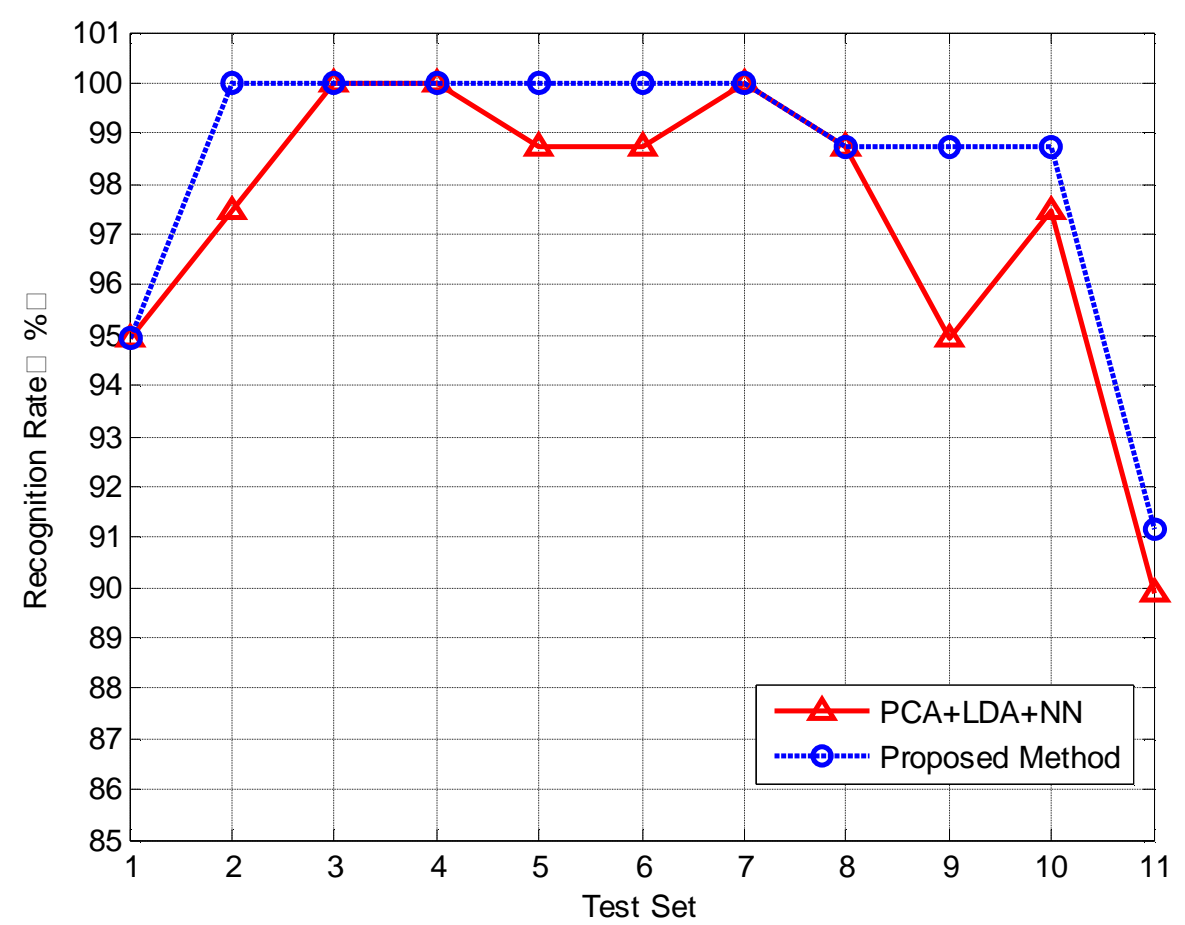

Fig. 3 The Recognition Rates for the USTB(79) ear database

\section{Conclusions}

In this paper, we proposed a new method for ear recognition using haar wavelet decomposition and LDA/GSVD. It is showed that the new method is better than PCA+LDA Under certain circumstances.

\section{Acknowledgment}

This work is supported by: (1) Project Supported by the Beijing Excellent Talent (Grant No.2012D005017000004). (2) Scientific Research Foundation of Beijing University of Civil Engineering and Architecture (Grant No. 00331611017).

\section{References}

[1]. A. Iannarelli, "Ear Identification", Forensic Identification Series, Paramont Publishing Company, 1989.

[2]. P.N Belhumeur, J.P.Hespanha, and D.J.Kriegman, Eigenfaces vs.Fisherfaces : Recognition Using Class Specific Linear Projection. IEEE Trans. Pattern Analysis and Machine Intelligence , vol.19, no.7, pp.711-720, 1997.

[3]. Peg Howland and Haesun Park, "Generalizing Discriminant Analysis Using the Generalized Singular Value Decomposition," IEEE Transactions on Pattern Analysis and Machine Intelligence, vol. 26, no. 8, August 2004:995-1006

[4]. I Daubechies and C Heil.'Ten Lectures on Wavelets", Society for Industrial and Applied Mathematics, 1992,6 (3):1671-1671

[5]. G.H. Golub and C.F. Van Loan, Matrix Computations, third ed. Johns Hopkins Univ. Press, 1996.:250-151 Baltimore and London 